\title{
Politische Ökologie: nicht-deterministische, globale und materielle Dimensionen von Natur/Gesellschaft-Verhältnissen
}

\author{
Sybille Bauriedl \\ Geographisches Institut, Universität Bonn, Bonn, Germany \\ Correspondence to: Sybille Bauriedl (bauriedl@uni-bonn.de)
}

Received: 7 June 2016 - Revised: 10 November 2016 - Accepted: 11 November 2016 - Published: 6 December 2016

\begin{abstract}
Kurzfassung. Political ecology is a research field comprising studies with a critical perspective on human/nature-relations - critical in both a political and an epistemological sense. Fundamental questions of political ecology, here, are related to just and equal access to resources, their contribution and control, and to the regimes of regulation. The article specifies the empirical and epistemological approaches within political ecology in the last decades. It does not tell a linear history or a single story, because political ecology emerges out of a continuous process of mutual inspirations of academic debates and activist practices. The research strands in political ecology operate with different ideas on how to conceptionalize nature: as social product, technonature, hybrid, or as actant. These conceptualisations are related to different approaches of neo-Marxist and post-structural epistemology. This article discusses the present debate of political ecology in two steps. After introducing a broader perspective of what critique means in political ecology, it gives an account of the various approaches for analysis of both, geographies and materialities of uneven development. The early studies of political ecology explain human/nature-relations as socially produced, related to a Marxist understanding of historical materialism. In recent debates of political ecology, this approach was confronted with a new materialist thinking of more fluid interrelations between nature and non-nature; it also addresses postcolonial studies' claim to decentralize the perspectives on history and geography in order to understand new forms of connectivity of nature and culture.
\end{abstract}

\section{Einleitung}

Die Politische Ökologie ${ }^{1}$ ist ein dynamisches Forschungsfeld, das in den letzten drei Jahrzehnten maßgeblich von der (Human)Geographie geprägt wurde. Dabei sind Forschungsansätze und Perspektiven quer zu disziplinären Grenzen u.a. aus der Ethnologie, der Entwicklungsforschung, den Politikwissenschaften und der Geschlechterforschung eingeflossen. Diese interdisziplinäre Mensch/Umwelt-Forschung hat zahlreiche englischsprachige Monographien, Sammelbände und Lehrbücher hervorgebracht (Bryant und Bailey, 1997; Keil et al., 1998; Robbins, 2004; Neumann, 2005; Peet et

\footnotetext{
${ }^{1}$ In diesem Beitrag wird „Politische Ökologie“ mit einem Großbuchstaben geschrieben, um ein spezifisches Forschungsfeld zu kennzeichnen.
}

al., 2010). Im Jahr 2015 sind gleich zwei weitere umfangreiche Handbücher zur Politischen Ökologie erschienen (Perreault und Bridge, 2015; Bryant, 2015). Anders als in der anglophonen Geographie ist die Politische Ökologie in den deutschsprachigen Ländern jedoch nie zum dominanten Ansatz der Umweltforschung oder zur Teildisziplin der Geographie geworden. Sie wird hier als eine spezifische Perspektive der sozialgeographischen Umweltforschung neben anderen, etwa der Vulnerabilitätsforschung (Bohle, 2007), der geographischen Risikoforschung (Bohle, 2001; Egner und Pott, 2010; Müller-Mahn, 2012) und der geographischen HazardForschung betrachtet (Felgentreff und Glade, 2008).

Ein Überblicksbeitrag kann die Vielfalt disziplinärer, nationaler und themenspezifischer Debatten der Politischen Ökologie nicht einholen. Die Politische Ökologie hat keine single story und lässt sich nicht als lineare Geschichte einer 
Forschungsrichtung nachzeichnen, da sowohl unterschiedliche erkenntnistheoretische Ansätze parallel verfolgt und weiterentwickelt als auch immer wieder neue umweltpolitische und umweltaktivistische Debatten aufgenommen wurden. Die folgende Darstellung der Politischen Ökologie versucht daher nicht, ein fixes Forschungsfeld, einen Literaturkanon oder eine Chronologie zu manifestieren. Sie soll vielmehr spezifische Analysepotentiale der Politischen Ökologie für die geographische Umwelt-, Entwicklungs- und Stadtforschung aufzeigen und umweltbezogene gesellschaftstheoretische Debatten nachzeichnen, die zur Bearbeitung komplexer Mensch/Natur-Dynamiken geeignet erschienen.

Meine Argumentation verfolgt zwei Diskussionsstränge der Politischen Ökologie. Der eine ist von gesellschaftstheoretischem Konsens geprägt, der andere eher von epistemologischem Dissens. Der erste Abschnitt stellt die Positionierung der Politischen Ökologie als kritische Mensch/UmweltForschung dar. Studien dieses Forschungsfeldes grenzen sich klar von funktionalistischen Prämissen der Umweltforschung und deren natur- sowie sozialdeterministischen Theorien ab. Die Politische Ökologie steht in Opposition zu deterministischen und monokausalen Erklärungsversuchen von Umweltkrisen, die die Komplexität von Herrschaftsverhältnissen außer Acht lassen. Sie stellt vielmehr Fragen nach dem Zugang zu, dem Ausschluss von und der Kontrolle über natürliche Ressourcen innerhalb struktureller Hierarchien und globaler Machtkonstellationen in den Mittelpunkt und nimmt Bezug auf lokale und transnationale Kämpfe emanzipatorischer Bewegungen. Der zweite Abschnitt setzt sich mit Forschungsperspektiven der Politischen Ökologie auseinander, die aktuell kontrovers diskutiert werden: Erstens mit verschiedenen Perspektiven auf globale Ungleichheit einer Third World Ecology, einer Global Political Ecology und postkolonialer Theorien, zweitens mit Perspektiven auf die Handlungsfähigkeit von Natur im historischen Materialismus und dem New materialism. Abschließend diskutiere ich erkenntnistheoretische Potentiale für Fragen globaler Umweltveränderungen und lokaler Umweltkonflikte, die sich aus der Verbindung neomarxistischer und poststrukturalistischer Ansätze für die Politische Ökologie ableiten lassen.

Meine Beobachtungen und meine eigene Beteiligung an diesen Debatten sind geprägt von diskursanalytischen und akteurszentrierten Studien zu nachhaltiger Stadtentwicklung, Klimaanpassung und Klimapolitik, Energiewende und Bioökonomie im europäischen, ostafrikanischen und internationalen Kontext. In diesen Feldern zeigt sich, dass monokausale und deterministische Verständnisse von Mensch/NaturVerhältnissen und darauf beruhende Problemlösungen soziale und globale Ungleichheit in den letzten Jahrzehnten verschärft haben. Die sozialen Kosten von Umweltveränderungen wie von Umweltschutzmaßnahmen werden weiterhin in den Globalen Süden (vgl. Emissionshandel) und ökologische Risiken in die Zukunft (vgl. Bioökonomie) verlagert (Bauriedl, 2015b). Damit hat die Notwendigkeit, herrschaftskritische Forschungsansätze weiterzuentwickeln und Umweltge- rechtigkeitsfragen zu bearbeiten, aus meiner Sicht nach wie vor hohe Relevanz.

\section{Politische Ökologie als kritische Mensch/Umwelt-Forschung}

Die Politische Ökologie lässt sich von anderen Ansätzen der geographischen Umweltforschung durch einen explizit herrschaftsanalytischen Zugang abgrenzen. Sie versteht „Umwelt" als ein dialektisches Verhältnis von Gesellschaft und Natur, das entlang von Ungleichheitsstrukturen organisiert ist. Untersuchungsgegenstände sind die Praktiken der Vermittlung von Gesellschaft und Natur, die sich u.a. in spezifischen Deutungen von Umweltproblemen, Inwertsetzung von Ressourcen, Aneignungen von Natur und Regulationsweisen gesellschaftlicher Naturverhältnisse zeigen. Die Politische Ökologie betrachtet Ressourcenkonflikte nicht allein als Ergebnis biophysikalischer Prozesse oder demographischer Dynamiken, sondern immer auch als Folge gesellschaftlicher Praxis, die sich in kapitalistischen Wohlstandsgesellschaften in Form von Überproduktion, Überkonsumption, Vergesellschaftung von Umweltkosten und der Reglementierung des Ressourcenzugangs zeigt. Gemeinsamer Ausgangspunkt dieser Perspektive auf Umweltfragen ist die Beobachtung sozialer und globaler Ungleichheitsverhältnisse. Diese wurden in der geographischen Politischen Ökologie insbesondere mit handlungstheoretisch, akteurstheoretisch und diskurstheoretisch ausgerichteten Analysen bearbeitet (Krings und Müller, 2001).

VertreterInnen der Politischen Ökologie kritisieren die Machtblindheit der meisten Ansätze der Umweltforschung. Gemeinsames Merkmal der Politischen Ökologie ist eine herrschaftskritische Perspektive, die Ben Wisner z.B. der Humanökologie, die in den 1970er Jahren die sozialgeographische Umweltforschung geprägt hat, abspricht. Diese bezeichnet er als eine ,unmenschliche“ Analyse von Umweltkrisen im Globalen Süden (Wisner, 2015:55), da sie eine sozial differenzierte Betrachtung ignoriert und damit eine gesellschaftskritische Analyse vernachlässigt. Studien der Politischen Ökologie zu Dürre, Klimawandel oder Fischsterben nehmen hingegen explizit die politischen und strukturellen Bedingungen dieser sozial-ökologischen Phänomene in den Blick, die u.a. durch Marktmechanismen, Eigentumsregime, staatliche Verwaltung, Rechtssysteme, wissenschaftliche Paradigmen, gewaltförmige Aneignungsstrategien, Emanzipationsbewegungen und deren lokale und globale Verankerung geprägt sind. Der Anspruch an eine sozialwissenschaftliche Umweltforschung, Herrschaftsverhältnisse in den Blick zu nehmen, durchzieht die Politische Ökologie bis heute. Wenn Studien der Politischen Ökologie z.B. auf die „,non-political politics of climate change“" (Swyngedouw, 2013) hinweisen und eine (Re-)Politisierung der Umweltpolitik fordern, dann soll damit nicht ausgedrückt werden, dass diese keine politische Relevanz oder Wirksamkeit hätte; angesprochen 
wird hiermit vielmehr die notwendige Reflexion impliziter Machtverhältnisse. Um die Herrschaftsförmigkeit von Umweltpolitik - und auch der Umweltforschung - zu erkennen, ist die behauptete Alternativlosigkeit der dominanten Umwelt- und Klimaschutzstrategien zu überprüfen. Der herrschaftskritische Ansatz der Politischen Ökologie ist in den meisten Studien mit den folgenden drei Beobachtungen verbunden: (1) Umweltkonflikte sind Ausdruck sozialer Krisen, (2) negative Folgen von Umweltveränderungen sind ungerecht verteilt, und (3) Umweltwissen ist ein politisch umkämpftes Feld. Diese Thesen kritisieren nicht nur die dominante Umwelt- und Klimapolitik, sondern auch den Mainstream der Umweltforschung, der bevorzugt systemkonforme Problemdeutungen vorlegt. Die daraus abgeleiteten Lösungsvorschläge zielen auf eine Optimierung aktuell nichtnachhaltiger Stoffströme, auf eine steigende Ressourcenproduktivität und eine Effizienzrevolution, die durch soziotechnische Lösungen und ökonomische Anreize eingeleitet werden sollen (vgl. „Faktor Vier“ des Wuppertal-Instituts), oder sie setzen auf ein vernunftbasiertes Konsumverhalten (vgl. UNESCO-Weltaktionsprogramm „Bildung für nachhaltige Entwicklung"). Diese Strategien werden gerahmt von dem Ideal einer ökologischen Modernisierung (vgl. Studien zur „Sozial-ökologischen Transformation“ des Wissenschaftlichen Beirats der Bundesregierung Globale Umweltveränderungen $a b$ 2011). Das ressourcenintensive Konsumund Wirtschaftsmodell der Industrieländer wird mit diesem technologieoptimistischen Entwicklungsideal nicht in Frage gestellt, und Ungleichheitsstrukturen werden zementiert (Bauriedl, 2015a).

Die folgenden zwei Abschnitte erläutern den gesellschaftspolitischen Kontext und das erkenntnisleitende Umweltverständnis der Politischen Ökologie, die zu einer fundamentalen Kritik an einer systemstabilisierenden sozialökologischen Transformation führen.

\subsection{Herrschaftskritik als erkenntnisleitende Perspektive auf Umweltkrisen und Umweltgerechtigkeit}

Die Politische Ökologie war von Beginn an mit Gerechtigkeitsfragen verbunden, die von sozialen und Umweltbewegungen initiiert wurden. Für die ersten Studien der Politischen Ökologie in Deutschland waren die Anti-AKWBewegung und die ,Waldsterben“-Diskussion genauso prägend wie Emanzipationsdebatten der Studenten-, Frauenund Jugendbewegungen. Im nordamerikanischen Kontext spielten Fragen der Umweltgerechtigkeit in Städten eine noch größere Rolle, da dort der Zusammenhang von Hautfarbe und Gesundheitsbelastung durch Industrieabfälle sehr deutlich war und Schwarze Bürgerrechtsbewegungen die rassistische Form sozialer Segregation in Stadtteilen mit akuter Umweltverschmutzung thematisierten. Der Bezug auf Kämpfe sozialer und Umweltbewegungen hat bis heute großen Einfluss auf die Formulierung wissenschaftlicher Fragestellungen der Politischen Ökologie.
Wichtige konzeptionelle Ansätze zur Betrachtung von Natur/Gesellschaft-Verhältnissen liefert seit den 1970er Jahren die Kritische Theorie der Frankfurter Schule u.a. mit Hans Magnus Enzensbergers Aufsatz ,Zur Kritik der politischen Ökologie“ (Enzensberger, 1974). ${ }^{2}$ Enzensberger fragte hierin, wann es zur Umweltkrise komme und welcher Faktor ausschlaggebend sei und wies nach, dass bei der Beantwortung dieser Fragen erstens die Klassenfrage vernachlässigt und zweitens die zunehmende Bevölkerungszahl überbewertet wurde (vgl. Abschnitt 2.2). Schon Theodor W. Adorno und Max Horkheimer hatten in ihrer ,Dialektik der Aufklärung“ den Willen zur Beherrschung der Natur mittels technisch-instrumenteller Rationalität als zentrales Element der Aufklärung beschrieben (Adorno und Horkheimer, 1947). Sie entwarfen außerdem den Begriff der „Nichtidentität von Natur“", mit dem sie die besonderen Qualitäten und die Eigengesetzlichkeit von Natur herausstellten und dazu aufforderten, diese unabhängig von einer kapitalistischen Produktion und Verwertung von Natur zu betrachten. Diese Perspektive der Frankfurter Schule, die das dialektische Verhältnis natürlicher und sozialer Prozesse betont, wurde insbesondere durch das 1989 gegründete Institut für sozialökologische Forschung (ISOE) in Frankfurt am Main mit dem Begriff der ,gesellschaftlichen Naturverhältnisse“ weiterentwickelt (Görg, 1999; Becker und Jahn, 2006). In dieser erkenntnistheoretischen Tradition stehen in der deutschsprachigen Politischen Ökologie Fragen nach der Regulation gesellschaftlicher Naturverhältnisse stärker im Fokus als in der anglophonen Debatte. Außerdem wird die deutschsprachige Politische Ökologie stärker von PolitikwissenschaftlerInnen getragen, die sich mit staats-, demokratie- und hegemonietheoretischen Fragen beschäftigen und sich orientiert an Antonio Gramsci mit den permanenten politischen, kulturellen sowie ideologischen Kämpfen um Hegemonie zwischen Staat und Zivilgesellschaft auseinandersetzen (vgl. Görg, 2003; Brand und Wissen, 2011b).

\subsection{Kritik an deterministischen und monokausalen Theorien}

Aus Sicht der Politischen Ökologie sind weder die Ursachen von Ressourcenknappheit noch von Naturkatastrophen allein mit natürlichen Prozessen zu erklären; beide Phänomene werden erst durch gesellschaftliche Verhältnisse relevant. Schon seit vorindustrieller Zeit macht die Aneignung von Naturressourcen (Agrarland, fossile Brennstoffe, Mineralien), die exzessive Nutzung der bisher als unendlich betrachteten Natur (Luft, Wasser, Sand usw.) und deren „Gratisdienstleistungen“ (Pflanzenbestäubung, Kohlenstoffabsorption usw.) immer weitere Teile der Natur zum knappen Gut. Die wissenschaftliche Aufmerksamkeit für Ressourcen-

\footnotetext{
${ }^{2}$ Erik R. Wolf hatte bereits 1972 den Begriff ,political ecology“ mit einer Arbeit zu Landkonflikten in Lateinamerika in die englischsprachige wissenschaftliche Debatte eingeführt (Wolf, 1972).
} 
knappheit hat wiederholt zur Diskussion um natürliche Grenzen gesellschaftlicher Entwicklung geführt. Der vielzitierte „Nature“-Artikel „A safe operating space for humanity“ („Ein sicherer Handlungsraum für die Menschheit“, Übers. S.B.) (Rockström et al., 2009) hat eine erneute Debatte um ökologische Rahmensetzungen gesellschaftlichen Handelns ausgelöst und den Begriff der ,planetarischen Grenzen“ ins Spiel gebracht. Mit dem Begriff des Planetarischen wird eine kollektive Verantwortlichkeit gegenüber einer universellen Gefahr hergestellt. Das Umweltproblem ist jedoch kein Menschheitsproblem, sondern trifft Menschen entlang von Armutsverhältnissen, Klassenverhältnissen und Geschlechterverhältnissen in unterschiedlicher Weise. Der Begriff der Grenze suggeriert außerdem, dass jenseits von ihr keine für den Planeten relevante Natur existiere (Görg, 2016:239). Die Politische Ökologie kritisiert genau diese Universalisierung und Differenzproduktion und betrachtet Grenzen der ökologischen Tragfähigkeit der Erde als sozial konstruiert und politisch umkämpft.

Eine naturdeterministische Definition von Ressourcenknappheit sowie die daraus abgeleiteten Praktiken (technologischer Umweltschutz, Substitution fossiler Energieträger usw.) entpolitisiert die Ursachen von ungleichem Ressourcenzugang und ungerechter Ressourcenverteilung, indem sie ihr zu Grunde liegende Machtverhältnisse unsichtbar macht (Braun, 2015a:102). Damit werden soziale Phänomene wie Armut oder Verwundbarkeit naturalisiert. Aus Sicht der Politischen Ökologie werden der Wert, die Nachfrage und die Verfügungsgewalt über eine Ressource gesellschaftlich verhandelt und institutionell festgelegt. Ressourcenkonflikte sind demnach der materielle Ausdruck von Kontroll- und Zielkonflikten dieser umkämpften Ressourcenverteilung. Aus dieser Perspektive wird beispielsweise der Mangel an sauberem Trinkwasser als Ergebnis sozialräumlicher Ungleichheit erkennbar, die sich z.B. in vielen Megastädten des Globalen Südens zeigt: eine gesicherte Trinkwasserinfrastruktur ist wohlhabenden Stadtteilen vorbehalten, und ärmeren Stadtteilen wird durch eine Privatisierung der Wasserversorgung die autonom organisierte Infrastruktur entzogen (Swyngedouw, 2004:4).

Betrachtet man den Klimawandel als Umweltkrise und nicht als Krise gesellschaftlicher Naturverhältnisse, so kann er als Problem einer aus dem Gleichgewicht geratenen externen „Natur“ beschrieben werden, das außerhalb gesellschaftlicher Verhältnisse existiert. Mit dieser Lesart des Klimawandels stehen z.B. klimabeeinflussende fossile Produktionsweisen und nicht-nachhaltiger Konsum nicht als krisenhaft zur Debatte. In der Politischen Ökologie wird „Natur“ nicht als externe Kategorie im Sinne einer von menschlichem Handeln und sozialen Strukturen unabhängige Materie verstanden. Auch zwanzig Jahre nach dem cultural turn ist diese Kritik an naturdeterministischen Argumentationen noch kein Konsens innerhalb der Geographie. Auch in der geographischen Umweltforschung wird „Natur“ in vielen Studien nach wie vor als Naturraum konzipiert, in dem das Soziale statt- findet und Lebensgrundlagen zur Verfügung gestellt werden. Auf diese Weise wird ihr eine Passivität zugeschrieben, die sie zur nutzbaren Ressource macht (Flitner, 1998:89). Für eine Politische Ökologie sind die Ursachen von Ressourcenkonflikten jedoch nicht durch die spezifische Ausstattung eines Raumes mit natürlichen Ressourcen, sondern durch eine ökonomisch und politisch motivierte Ressourcenverknappung zu erklären.

Neben naturdeterministischen Argumenten finden sich in der Umweltforschung auch sozialdeterministische Erklärungszusammenhänge und sozialdarwinistische Umweltverständnisse, die in der Politischen Ökologie seit langem als apolitisch kritisiert werden (Peet, 1985). Umweltzerstörung und Umweltveränderungen werden in einer NeoMalthusianischen Lesart als Folge einer exponentiell zunehmenden Weltbevölkerung betrachtet, die als „Überbevölkerung" bezeichnet wird. Thomas Malthus hatte im 18. Jahrhundert prognostiziert, dass die Bevölkerung überproportional zur landwirtschaftlichen Produktion anwachsen werde. Diese Entwicklung nannte er „Bevölkerungsfalle“, da sie ein Hemmnis ökonomischen Wachstums sei (Malthus, 1789). Diese These steckt auch im Begriff „Bevölkerungsbombe“, mit dem der Zoologe und Biogeograph Paul Ehrlich globale Hungersnöte für die 1970er Jahre prognostizierte (Ehrlich, 1968). Das Überbevölkerungsnarrativ stellt einen Zusammenhang zwischen hoher Geburtenrate (explizit im Globalen Süden), globaler Umweltzerstörung und Entwicklungsproblemen her (vgl. Meadows et al., 1972). Die USA brachten Anfang der 1970er Jahre den Vorschlag einer Geburtenkontrolle in der sogenannten Dritten Welt in die Diskussion zum globalen Umweltschutz. Sozialdeterministische Argumentationen liegen auch heute noch einigen Studien der Entwicklungsforschung zugrunde, die den Waldrückgang in tropischen Ländern mit der Praxis der Brandrodung durch Kleinbauern erklären, was mit dem Bevölkerungswachstum zu zunehmendem Waldverlust führe. Und auch in der internationalen Umwelt- und Klimapolitik wird der Handlungsdruck immer wieder mit der wachsenden Weltbevölkerung als zentrales Problem globaler Umweltveränderungen begründet. Sozialdeterministische Argumente stützen Modernisierungsmodelle, die die Problemlösungsfähigkeit der Marktwirtschaft und die Produktivitätssteigerungspotentiale einer industriellen Landwirtschaft betonen.

Die Politische Ökologie nimmt ökologische und demographische Dynamiken durchaus in den Blick, hebt aber deren Wechselwirkung mit globalen gesellschaftlichen und ökonomischen Prozessen hervor. Auf diese Weise geraten gesellschaftliche Naturverhältnisse des Globalen Nordens (industrielle Landwirtschaft, Nutzung fossiler Energien usw.) und deren Auswirkungen auf den Globalen Süden in den Fokus der Problemursachenanalyse. Michael Watts hatte mit seiner Studie „Silent Violence“ zur Ernährungskrise in Nigeria (Watts, 1983) den critical turn in der geographischen Umweltforschung eingeläutet, indem er Dürre und Hunger in Westafrika als gesellschaftlich und politisch verursacht er- 
klärte und nachwies, dass die auf globale Märkte ausgerichtete Klientelwirtschaft politischer Eliten größeren Einfluss auf diese „Naturkatastrophe“ haben als die wachsende Bevölkerungszahl oder rückständige Technologien von Kleinbauern und -bäuerinnen.

\section{Forschungsperspektiven der Politischen Ökologie}

Wie lässt sich die herrschaftskritische Perspektive der Politischen Ökologie in herrschaftssensible Analysen übertragen? Welche Kategorien von Herrschaft werden dabei berücksichtigt? Die Politische Ökologie nutzt eine große Breite erkenntnistheoretischer Ansätze, die in den verschiedenen nationalen Debatten und über die letzten Jahrzehnte mit unterschiedlicher Aufmerksamkeit verfolgt wurden. Während sich die frühen Studien an strukturalistischen Ansätzen und einer neomarxistisch ausgerichteten politischen Ökonomie orientierten und einen Fokus auf regionale Ressourcenkonflikte im Kontext internationaler Ressourcenregime legten, wurden ab den 1990er Jahren poststrukturalistische Ansätze und relationale Theorien aufgenommen und in den letzten Jahren verstärkt die Handlungsfähigkeit des Nicht-Menschlichen berücksichtigt. Die folgenden zwei Abschnitte fassen die Breite der erkenntnistheoretischen Debatten der Politischen Ökologie zu Globalität und Materialität mit Bezug auf neomarxistische und poststrukturalistische Ansätze zusammen.

\subsection{Geographien der Politischen Ökologie: Nord/Süd-Perspektiven, globale und postkoloniale Perspektiven}

Die frühen Studien der Politischen Ökologie setzten sich in den 1980er Jahren mit Problemen der Bodendegradation, Erosion und Ressourcenextraktion in Asien, Afrika und Südamerika auseinander. Sie erklärten diese Probleme nicht mehr allein als Ergebnis biophysikalischer Phänomene, lokaler Kämpfe um knappe Ressourcen oder der Übernutzung regenerativer Ressourcen. Vielmehr werden Erklärungsansätze für eine ungleiche Entwicklung in Ländern des Globalen Nordens und des Globalen Südens gesucht. Piers Blaikies Studie ,The Political Economy of Soil Erosion in Developing Countries“ (Blaikie, 1985) wird oft als Pionierarbeit einer neomarxistisch Entwicklungskritik und als Grundstein der Politischen Ökologie beschrieben. Die Politische Ökologie gründet nicht auf der Arbeit einzelner WissenschaftlerInnen. Blaikies Studie zur Degradation von Landwirtschaftsflächen in Nepal ist jedoch bis heute eine wichtige Referenzstudie. Er stellt darin die Interpretation von Bodenerosion allein als Folge von Bevölkerungsdruck und Kommerzialisierung der Landwirtschaft in Frage und erklärt die Ursache von Armut und sozialer Verwundbarkeit sowohl mit Blick auf die hierarchische Gesellschaftsordnung in Nepal als auch durch eine vom Globalen Norden dominierte politische Ökonomie. Es folgten ähnliche Studien, die etablierte Erklärungsmuster von „Unterentwicklung“ und Umweltzerstörung reinterpre- tierten (vgl. Fairhead und Leach, 1996) und damit die Entwicklungskapazität von Kleinbauern hervorhoben sowie die Folgen von Strukturanpassungsprogrammen und die erzwungene Öffnung nationaler Agrarmärkte im Globalen Süden problematisierten. Bis Ende der 1990er Jahre konzentrierten sich Studien der Politischen Ökologie auf Mikropolitiken, insbesondere auf die Kämpfe von Kleinbauern im Globalen Süden und deren Zugang zu den für sie relevanten Produktionsmittel (Land, Wasser, Saatgut), zu Bildung und Wissen (entitlement) und ihren Einfluss auf Entscheidungsprozesse (empowerment).

Lokale Umweltprobleme im Globalen Süden wurden mit Bezug auf neomarxistische Entwicklungstheorien und Weltsystemtheorien als Ergebnisse der Integration peripherer Weltregionen in den kapitalistischen Weltmarkt erklärt (Dietz und Engel, 2011). Dabei wurden Marginalisierungsund Ungleichheitsstrukturen nicht als unbeabsichtigte $\mathrm{Ne}$ benfolgen, sondern als inhärenter Bestandteil dieses Systems betrachtet. Neil Smith zeigte in seinem Buch „Uneven Development. Nature, Capital and the Production of Space“ (Smith, 1984) mit Hilfe von Marx' Werttheorie, in welcher Weise das Verhältnis von Natur und Gesellschaft in modernen Industriegesellschaften über kapitalistische Produktionsbedingungen vermittelt wird. Die historisch spezifische Gesellschaftsform des Kapitalismus basiert auf einer permanenten Einverleibung von Arbeitskraft und Ressourcen, die soziale und globale Ungleichheit produziert. Raymond Bryant und Sinéad Bailey benannten diesen Fokus auf Geographien der Ungleichheit aus einer Nord/Süd-Perspektive ,Third World Political Ecology“ (Bryant und Bailey, 1997). In ihrem gleichnamigen Sammelband zeigen sie anhand zahlreicher Beispielstudien die ökonomischen Verflechtungen lokaler Ressourcenkämpfe in Entwicklungsländern im Kontext globaler Arbeitsteilung und Ressourcenaneignung. Ausgangspunkt dieser Studien sind Analysen zu den Akteurskonstellationen, die differenziert werden in ortsbezogene Akteure (place-based actors) wie Kleinbauern, Fischer, Tierzüchter, und nicht ortsbezogene Akteure (non-place-based actors) wie internationale Unternehmen, Finanzdienstleister, internationale Institutionen und nationale Entscheidungsträger. In Abgrenzung zu diesem Entwicklungsländerfokus hatten Richard Peet und Michael Watts schon in ihrem ersten Sammelband „Liberation Ecology“ eine größere Aufmerksamkeit der Politischen Ökologie für die Verflechtungen lokaler Gemeinschaften mit globalen Kräften eingefordert, die über eine Nord/Süd-Perspektive hinausgeht (Peet und Watts, 1996). Diesen Anspruch manifestieren sie mit dem Buchtitel „Global Political Ecology“ ihres jüngsten Sammelbandes (Peet et al., 2010). Sie plädieren für ein ,theorizing up“ mit dem Argument, dass die Debatten und Praktiken des Stadtgärtnerns in europäischen Großstädten wie auch die Strategien internationaler Klimapolitik von den gleichen Umweltdiskursen gerahmt werden. Nicht mehr allein ,place-basedactors" sollen im Zentrum der Politischen Ökologie stehen, 
sondern ein systematischer Vergleich lokaler Umweltkrisen und -konflikte im Kontext globaler Politiken.

Nach dem Ende der bipolaren Weltpolitik formierte sich seit den 1990er Jahren eine globale Umweltpolitik, die von der Hoffnung auf kooperativ arbeitende politische Institutionen getragen wurde (global environmental governance). Das Verhältnis von Demokratie, Markt, Staat und Umwelt wurde im Kontext der ökonomischen Globalisierung von diesem Zeitpunkt an neu definiert. Nicht mehr ordnungsrechtliche Steuerung, sondern deregulierte wirtschaftliche Aktivitäten und marktgerechte Instrumente wurden zum Mantra internationaler Umweltpolitik. NaturwissenschaftlerInnen ist über ihre Beteiligung in UN-Institutionen und Verhandlungen bei der Legitimierung neoliberaler Umweltpolitik eine immer größere Bedeutung zugekommen. Es gäbe keine Karbonökonomie ohne den Wissenschaftsdiskurs zu planetarischen Grenzen und $\mathrm{CO}_{2}$-Monitoring. Es gäbe keine ökonomische Inwertsetzung von Ökosystemdienstleistungen ohne das Millennium Ecosystem Assessment der UNUmweltorganisation (MEA, 2005). Und es gäbe keine Vision einer Grünen Ökonomie ohne die Behauptung von ÖkologInnen und ÖkonomInnen, dass eine langfristige Entkopplung von Wirtschaftswachstum und Umweltverbrauch möglich sei. Das damit verbundene Wachstums- und Wohlstandsversprechen für alle ist die zentrale Herrschaftsstrategie industriekapitalistischer Regime. In dieser Macht/WissenFormation internationaler staatlicher und wissenschaftlicher Organisationen wird Natur als reparierbar vermittelt (Leach et al., 2012). Das von Geologen und Meteorologen gemeinsam ausgerufene Anthropozän (Steffen et al., 2007) erscheint als globaler Unfall eines industriekapitalistischen Experiments, das mit Technologien repariert werden kann, die fossile Energieträger ersetzen und der Atmosphäre Kohlenstoff entziehen.

Mit dem Bedeutungsgewinn nicht-staatlicher Akteure und der Einführung marktförmiger Instrumente in der internationalen Umweltpolitik Ende der 1990er Jahre hat sich eine neue Form der Umwelt-Gouvernementalität herausgebildet. Deren Kritik führte zu einer enormen Produktivität der Politischen Ökologie, so dass Michael Watts sogar von einer „New Political Ecology“ sprach (Watts, 2000), die sich mit umweltpolitischen Diskursen wie „Nachhaltigkeit“ und später auch „Grüner Ökonomie“ und „Grüner Entwicklung“ auseinandersetzt. Die Politische Ökologie untersuchte nun verstärkt die umkämpfte kulturelle Praxis der Umweltpolitik. Mit der marktorientierten internationalen Klimapolitik und den Akkumulations- und Verwertungsinteressen einer Grünen Ökonomie sind jedoch in den letzten Jahren gleichzeitig Nord/Süd-Perspektiven wieder zurück in den Fokus der Politischen Ökologie gerückt. David Harvey spricht von einer Enteignung öffentlicher Güter im Globalen Süden für private Gewinninteressen im Globalen Norden (accumulation by dispossession), die zu einem neuen Imperialismus geführt haben (Harvey, 2004). Diese Form der Aneignung beinhaltet den Transfer von Eigentum bzw. das Nutzungsrecht und die
Kontrolle über öffentlich zugängliche Ressourcen. In Afrika, Südostasien und Südamerika war im letzten Jahrzehnt eine Landnahme durch internationale InvestorInnen für den Anbau von Pflanzen zur Agrartreibstoffproduktion (Zuckerrohr, Palmöl, Jatropha) im großen Maßstab zu beobachten.

In den zahlreichen Studien der Politischen Ökologie zu Fragen der Landnahme für Agrartreibstoffe und für Kohlenstoffsenken ${ }^{3}$ sehen James Fairhead, Melissa Leach und Ian Scoones die These der Akkumulation durch Enteignung bestätigt. Sie nennen diese spezifische Form der Landnahme mit Instrumenten des globalen Umwelt- und Klimaschutzes green grabbing (Fairhead et al., 2012:241), da sich seit der Einführung des staatlich regulierten und privat organisierten Emissionszertifikatehandels eine zusätzliche Dynamik entfaltet hat. Auch die staatliche Entwicklungshilfe wird in Projekte kanalisiert, die einen zusätzlichen Nutzen für den globalen Klimaschutz generieren sollen. Industrieländer profitieren hierbei doppelt, indem sie ihre Klimawandelverantwortung vergesellschaften und durch den Einsatz von Klimaschutztechnologien (Windkraft, Geothermie, Wasserkraft) ihre Ziele einer Grünen Ökonomie verfolgen können (vgl. McCarthy und Prudham, 2004). Heidi Bachram nennt diese Form des globalen Klimaschutzes „Carbon colonialism“" (Bachram, 2004), da die Verlagerung der Klimaschutzmaßnahmen in Entwicklungsländer und die Möglichkeit der Industrieländer, Verschmutzungsrechte zu kaufen, kolonialen Herrschaftsverhältnissen folgt.

Anders als die Studien einer Third World Ecology, beschäftigen sich Studien, die sich kritisch mit dem Terminus „Grüne Ökonomie“ auseinandersetzen, mit sozialen Verhältnissen jenseits des Globalen Südens. Ulrich Brand und Markus Wissen haben den Begriff der ,imperialen Lebensweisen" eingeführt, mit dem sie die Wohlstands- und Konsumgesellschaften des Globalen Norden in den Fokus rücken und die regulationstheoretische Kategorie der „Entwicklungsweise" für Industrieländer anwenden (Brand und Wissen, 2011a). Sie bezeichnen damit eine Lebensweise, die auf Grund ihrer extensiven Inanspruchnahme ökologischer Ressourcen und der Globalisierung von Umweltverschmutzung nicht verallgemeinerbar ist. Der imperiale Charakter dieser Lebensweise zeigt sich darin, dass sie auf eine Peripherie angewiesen ist, deren Ressourcen, Arbeitskräfte und Senken sie sich aneignet. Die Ausweitung einer imperialen Lebensweise sehen Brand und Wissen als Ursache anhaltender multipler Krisen (ebd.). Trotz der Krise des Fordismus und der politischen Bedeutung der Umweltbewegungen seit den 1970er Jahren hat sich eine imperiale Lebensweise in den Industriestaaten erhalten. Mit der neoliberalen Globalisierung seit den

\footnotetext{
${ }^{3}$ Das klimapolitische Instrument des Waldschutzes und der Ausweitung von Waldflächen (REDD+) soll die Speicherung und Aufnahme von Kohlenstoff fördern. Waldschutzmaßnahmen in Entwicklungsländern können über ein standardisiertes Zertifizierungsund Monitoringverfahren mittels Kohlenstoffzertifikaten finanziert werden.
} 
1990er Jahren hat der Ressourcenanspruch von Konsumgesellschaften sogar noch zugenommen und sich auf die neuen Schwellenländer ausgebreitet.

Diese Verschiebung des analytischen Blicks in Richtung des Globalen Nordens ist auch eine Reaktion auf die Aufforderung der postkolonialen Studien, die Zentralität des Wissens und ein europäisches Entwicklungsideal in Frage zu stellen. In der Umwelt- und Entwicklungsforschung ist immer noch Europa der stille, privilegierte Referenzpunkt. Auch die Politische Ökologie mit einem herrschaftskritischen Anspruch ist noch weit entfernt davon, Wissenstraditionen $\mathrm{zu}$ dekolonisieren und sich vom dualistischen Denken der Moderne zu lösen. Auf forschungspraktischer Ebene gibt es Versuche, der Forderung Europa zu provinzialisieren (Chakrabarty, 2000) z.B. durch kollaborative Studien $\mathrm{zu}$ entsprechen, die ExpertInnen unterschiedlicher lokaler und kultureller Kontexte verbinden und eine dekoloniale Forschungsethik verfolgen. Matthew Turner plädiert außerdem dafür, dass gerade im Kontext von lokalen Konflikten ProtagonistInnen der Politischen Ökologie sich aktiv an Gerechtigkeitsfragen beteiligen sollten (Turner, 2014:480). In ähnlicher Weise sprach sich Tim Forsysth für eine ,situated environment science“ aus, die sich an konkreter Praxis lokaler Auseinandersetzungen beteiligt (Forsyth, 2011).

Ein wichtiger Ansatz zur postkolonialen Debatte ist für die Politische Ökologie die Konzeption einer globalen Kolonialität. Diese versteht u.a. Orte, Land und Körper als verwoben in Akkumulationsprozessen, die mit Gewalt verbunden sind, aber auch Widerstand hervorbringen (Mignolo, 2011). Dieser Ansatz geht weit über eine kapitalismus- oder neoliberalismuskritische Perspektive hinaus und versteht die koloniale Machtmatrix als ein europäisch-modernes, kapitalistischpartriarchales System (Grosfoguel, 2007:218). Die postkoloniale Geographie und auch die postkolonialen Ansätze in der Politischen Ökologie stellen kein einheitliches Forschungsfeld dar. Die Auseinandersetzung mit Kolonialismus als räumlichem Prozess der Gegenwart (vgl. Post-development und Subalternity studies) und die Dekonstruktion diskursiver Kontinuität (vgl. Postcolonial studies) stehen oft unverbunden nebeneinander. Beide Perspektiven verfolgen jedoch den utopischen Anspruch einer dezentrierten Wissenschaft und nehmen eine oppositionelle, gegenhegemoniale Haltung gegenüber der symbolischen und materiellen Manifestation von Ungleichheit und Marginalisierung postkolonialer Subjekte und Wissensbestände als Ergebnis kultureller und rassistischer Differenz ein (Radcliffe, 2005:292).

Die Auseinandersetzung mit kolonialen Bedingungen geht in der Geographie über den sprachlichen und kulturellen Fokus der postkolonialen Studien hinaus und versucht diskursive Perspektiven mit materiellen Folgen globaler Ungleichheit zu verbinden (McEwan, 2003). Eine postkoloniale Politische Ökologie will nicht nur marginalisierte Subjekte zur Sprache bringen, sondern betrachtet auch Natur als marginalisiertes Quasi-Subjekt, um die ,,neuen postkolonialen Ökologien“ (Jackson, 2014) in den Blick zu rücken.

\subsection{Naturen der Politischen Ökologie: Ressource, Nicht-Mensch, Hybrid}

Um welche „Naturen“ geht es bei der Analyse von Mensch/Natur-Verhältnissen? Die Politische Ökologie - wie auch die sozialwissenschaftliche Umweltforschung im Allgemeinen - betrachtet unterschiedliche Formen von Materialität und Handlungsfähigkeit von Natur. Die erkenntnistheoretische Debatte bewegt sich dabei aktuell zwischen Ansätzen eines historischen Materialismus und eines New materialism. Ersterer ist nach wie vor forschungsleitend für eine Politische Ökologie, die sich an einem neomarxistischen Materialismusbegriff orientiert. Neomarxistische Ansätze betrachten eine durch Technik und Warenproduktion, durch Landwirtschaft und Umweltschutz unter kapitalistischen Bedingungen produzierte Natur als relevanten Forschungsgegenstand (vgl. Ekers und Loftus, 2012). Menschliche Aktivität wird in marxistischen Ansätzen als permanente UmweltGestaltung (environment-making) unter kapitalistischen Bedingungen verstanden (Moore, 2015).

Die Diagnose einer Geographie ungleicher Entwicklung als Effekt globaler Neoliberalisierung hat am Anfang des 20. Jahrhunderts zu einer enormen Produktivität in der Politischen Ökologie geführt (vgl. Harvey, 2006). Große Einigkeit herrscht darüber, dass der Umfang und die Reichweite der Neoliberalisierung seit Mitte der 1990er Jahre zugenommen haben. Die Politische Ökologie hat sich in dieser Phase intensiv mit Praktiken der Privatisierung und Kommodifizierung von Natur auseinandergesetzt. Die Autoren des Sammelbandes „Neoliberal Environments“ untersuchen hierzu den $\mathrm{Zu}$ sammenhang von Markt, Staat, Deregulation und Bewertung von Natur und kommen zu dem Schluss, dass eine Neoliberalisierung der Umweltpolitik unerwünschte Umweltveränderungen und eine Verschärfung sozialer Ungleichheit zur Folge haben (Heynen et al., 2007:2). Sie formulieren als Komponenten einer neoliberalen Agenda der Naturaneignung, dass erstens Ökosysteme durch eine Bepreisung zu handelbaren Gütern gemacht werden (Monetarisierung), dass zweitens Gemeinschaftsgüter wie Wasser, Land oder Tropenwald von staatlichen Institutionen an Unternehmen übereignet werden (Privatisierung) und dass drittens internationale und nationale Regierungsorganisationen kapitalistische Naturaneignung durch Entwicklungsprogramme legitimieren (Environmental Governance) (ebd.).

Die These einer Neoliberalisierung von Natur wird in der Politischen Ökologie kontrovers diskutiert. Die Frage, inwieweit eine neoliberalisierte Ökonomie zu einer neuen Qualität der Naturaneignung geführt hat oder ob diese ein inhärenter Bestandteil des Kapitalismus ist, wurde bisher nicht abschließend beantwortet. Noel Castree und Karen Bakker identifizierten als zentrale Herausforderungen für die Politische Ökologie eine differenziertere Analyse nicht nur unterschiedlicher Ziele und Taktiken der Neoliberalisierung, sondern auch unterschiedlicher Ressourcen auf Basis vergleichender Studien (Castree, 2008). Daraus ließe sich nicht nur 
eine differenzierte Betrachtung der Neoliberalisierung von Wald, Wasser, Land usw. ableiten, sondern auch Gründe für deren Scheitern oder Formen des Widerstands - auch der Natur - analysieren (Bakker, 2009). Nicht jeder Kleinbauer und jede Kleinbäuerin endet als neoliberal diszipliniertes Subjekt (Fairhead et al., 2012:247), und nicht jede Natur ist allein passiver Gegenstand politischer Ökonomie. Die erkenntnistheoretische Fokussierung des historischen Materialismus auf eine sozial produzierte Natur wurde in den letzten Jahren vielfach als kulturell universalistisch und ökonomisch reduktionistisch kritisiert, da für eine Neoliberalisierungsdiagnose allein die Materialität von Natur als Ressource im Vordergrund steht und die Lebendigkeit alles Nicht-Menschlichen wenig Beachtung findet (Bakker und Bridge, 2006).

Neomarxistische GeographInnen betrachten aktuell insbesondere in Schwellenländern eine von nationalstaatlichen Eliten und internationalen Konzernen vorangetriebene Aneignung globaler Natur als eine neue Phase ursprünglicher Akkumulation (Neo-Extraktivismus). Die sich ausbreitende Karbonökonomie zeigt, dass auch in einer post-fossilen Welt spezifische Formen der Transformation, Aneignung, Kontrolle von Natur konstitutiv bleiben für soziale Herrschaft. Der material turn der Politischen Ökologie kann vor dem Hintergrund dieser Diagnose eher als Fortbestand historischmaterialistischer Ansätze verstanden werden. Eine agency der Natur kommt höchstens als eine Form der Widerständigkeit von Ressourcen in Betracht, wenn Wasser sich nicht vollständig kommodifizieren oder sich der Kohlenstoffkreislauf nicht vollständig ökonomisieren lässt. Eine Aufmerksamkeit für die eigenständige Produktivität von Natur außerhalb, jenseits oder vor neoliberaler Inwertsetzung findet sich in diesen ressourcengeographischen Studien nicht (Braun, 2015b:5).

Dabei wird im Rahmen des cultural turn auch in der Geographie schon seit einem Jahrzehnt die rein gegenständliche Betrachtung von Materialität und materieller Erfahrung problematisiert. Mit diesem Perspektivenwechsel auf Natur verändert sich auch das Denken über Biodiversitätsschutz, Körper, Organismen, Raum und Transformation in der Politischen Ökologie (Braun, 2015a:3). Die damit verbundene erkenntnistheoretische Auseinandersetzung mit nicht-linearen Systemen und der Dingmacht von Natur wird bisher nur von einzelnen wenigen GeographInnen betrieben. Ein erweitertes Verständnis von agency hat Sarah Whatmore mit ihrem Konzept der „hybriden Geographien“ in die geographische Diskussion eingebracht (Whatmore, 2002). In ihrem Beitrag „Political Ecology in a More-than-Human World“ diskutiert sie die Bedeutung unterschiedlicher Wissenspraktiken und deren Naturverständnis für die Beurteilung von Hochwasserrisiken (Whatmore, 2013). Die Vorstellung einer agency von Natur in Form einer Intra-Aktivität zwischen Lebewesen unterschiedlicher Spezies hat Jamie Lorimer mit einer Studie zum Biodiversitätsschutz in die geographische Debatte getragen (Lorimer, 2010). Diese Studien beschäftigen sich dabei mit den Fragen: Wie kann Politik und Wandel gedacht wer- den, wenn eine Handlungsmacht (agency) von materiellen Dingen und Agenten ernst genommen wird? Welche naturund geodeterministischen Fallen sind mit dem Zugeständnis einer Handlungsfähigkeit von Natur verbunden? Und werden sozialkonstruktivistische Ansätze mit dem New materialism über Bord geworfen?

Die aus den Gender studies und den Science and technology studies inspirierten Bücher „Meeting the Universe Halfway“ der Physikerin Karen Barad (2007) und „Vibrant Matter" der Politikwissenschaftlerin Jane Bennett (2010) geben viel diskutierte Impulse für die Beantwortung dieser Fragen. Beide grenzen sich deutlich von einem anthropozentrisch ausgerichteten historischen Materialismus $\mathrm{ab}$, indem sie die Eigenschaften und Handlungsfähigkeiten von Materie berücksichtigen (vgl. Barad, 2003). Ausgangspunkt sind Beobachtungen vielfältiger handlungsmächtiger „Natur"-Phänomene wie dem Klimawandel, dem globalen Artensterben oder Pandemien und gleichzeitig einer neuen Fluidität von Mensch/Natur-Verhältnissen durch Medizintechnik, synthetische Biologie und digital beschleunigte Datenströmen. Mit einer immer engeren Verbindung von Mensch, Natur, Technik und Wissenschaft ist eine Neudefinition der etablierten Grenzziehung zwischen Menschlichem und Nicht-Menschlichem und der Vorstellung von Materialität virulent geworden. Die Modi der Erkundung und Konzeptualisierung materieller Realitäten sind jedoch weiterhin noch in den Anfängen (Coole und Frost, 2010). Dabei sollten sich erkenntnistheoretische Zugänge gegenseitig anregen. Bruce Braun sieht dieses Potential sowohl für das aus seiner Sicht reduktionistische Mensch/Natur-Verständnis des historischen Materialismus sowie auch für die aus seiner Sicht Geschichtsfremdheit der New materialism-Debatte. Eine Verbindung beider Perspektiven könnte dazu beitragen, die Neoliberalisierung von Natur nicht mehr allein mit der Gestaltungskraft des Kapitals zu untersuchen, sondern auch die Energien, Bedingungen und Effekte jenseits ökonomischer Inwertsetzung von Ökosystemdienstleistungen in den Blick zu nehmen (Braun, 2015b:2).

Dipesh Chakrabarty versteht diese nicht-deterministische Perspektive auf Mensch/Natur-Verhältnisse als eine zentrale Herausforderung sowohl für die Umweltforschung wie für die postkolonialen Studien: „The science of anthropogenic global warming has doubled the figure of the human - you have to think of the two figures of the human simultaneously: the human-human and the nonhuman-human" (2012:11). Chakrabarty argumentiert, dass sich postkoloniale Kritik und jegliches, politisches Denken mit den skalaren Veränderungen im Anthropozän und den Realitäten der globalen Erwärmung auseinandersetzen muss.

\section{Fazit}

Die Politische Ökologie versteht sich als engagierte Umweltforschung, die den Neutralitäts- und Objektivitätsan- 
spruch wissenschaftlicher Arbeit bei Umweltfragen für obsolet erklärt und der (Selbst-)Reflexion und Positionalität von wissenschaftlicher Wissensproduktion einen hohen Stellenwert einräumt. Die Vielstimmigkeit der Politischen Ökologie lässt immer wieder Ansätze zu, die neue Perspektiven einer herrschaftskritischen Problembetrachtung von Umweltkrisen, Umweltrisiken und Umweltkonflikten eröffnen. Dies ermöglicht es, alternative Naturverhältnisse jenseits von kapitalistischen Dogmen denkbar zu machen und in die Umweltforschung zu tragen. Die Beiträge der Politischen Ökologie münden jedoch nicht automatisch in anwendbaren Ergebnissen. Im Wissenschaftsverständnis der Politischen Ökologie müssen Lösungsvorschläge zur Bearbeitung von Umweltproblemen in geeigneten gesellschaftlichen Kontexten ausgehandelt werden, da sie weitreichende Aspekte sozialer und globaler Gerechtigkeit berühren. Die Suche nach herrschaftssensiblen Praktiken betrifft jedoch auch die Politische Ökologie selbst als Teil der gesellschaftlichen Institution Wissenschaft. Welche konkreten forschungspraktischen Konsequenzen folgen aus der Kritik an einer Umweltforschung, die Natur als passive Ressource kapitalistischer Inwertsetzung und Verwertung betrachtet und aus der neuen Aufmerksamkeit für die Vitalität des Nicht-Menschlichen? Wie kann Natur als Agent und als Quasi-Subjekt untersucht werden?

Der historische Materialismus als zentraler Ansatz der Politischen Ökologie zieht seine analytische Kraft aus einer kritischen Historiographie. Diese müsste sich von ihrem reflexhaften Verweis auf eine Universalgeschichte des Kapitalismus und eine Totalität neoliberaler Regulation lösen. Die fundamentale Systemkritik einer Politischen Ökologie, die Natur nur noch als ,environmental fix“ innerhalb von Akkumulationskrisen versteht, wird m.E. in der Konfrontation mit Ansätzen des New materialism und der postkolonialen Theorie an Relevanz für eine gesellschaftstheoretisch ausgerichtete Umweltforschung verlieren. Fragestellungen der sozialwissenschaftlichen Umweltforschung sollten stärker als bisher auf eine erkenntnistheoretische Dezentralisierung des menschlichen Akteurs im Mensch/Natur-Verhältnis (Morethan-human philosophies) und der Provinzialisierung eurozentrischer Perspektiven (Postcolonial philosophies) ausgerichtet sein. Wie sind Bedingungen von Kolonialismus und Globalisierung mit den Bedingungen globaler Umweltveränderungen verwoben? Wie können wir anders als in einer eurozentrischen Natur-Kultur-Trennung über globale Umweltveränderungen nachdenken? Wie kann der Mensch als Naturgestalter (ecological agent) unter kolonialen Bedingungen betrachtet werden?

Die Verbindung von historischem Materialismus, New materialism und postkolonialen Studien kann einen erkenntnistheoretischen Raum eröffnen, um eine nicht-kapitalistische, post-kapitalistische oder mehr als kapitalistische Welt vorstellbar zu machen, wenn sowohl historische Bedingtheit von Mensch/Natur-Verhältnissen wie auch eine Handlungsfähigkeit oder Intra-Aktivität von Natur berücksichtigt wird. In den aktuellen Debatten des cultural turn und des material turn sind diese Ansätze in die Geographie eingeflossen und werden die Perspektiven der Politischen Ökologie in den nächsten Jahren prägen. Die Politische Ökologie hat das Potential, Fragen zu den Bedingungen und den ökologischen und sozialen Folgen der Aneignung von Natur zu verbinden und damit zu einer dezentralisierten Perspektive auf Entwicklung, zu einer nicht-deterministischen Perspektive auf Natur und zu einer nicht-linearen Zukunftsvorstellung in der Umwelt-, Stadt- und Entwicklungspolitik sowie -forschung beizutragen.

Danksagung. In meine subjektive Erzählung der Politischen Ökologie sind vielfältige Diskussionen des Netzwerks Politische Ökologie, des Netzwerks Feministische Geographien des New materialism, des Projektseminars „Globale Umweltveränderungen und Politische Ökologie“ im Wintersemester 2015/16 am Geographischen Institut der Universität Bonn und des Workshops „Kritische Umweltforschung“ am Institut für Geographie der Universität Hamburg am 15. Oktober 2016 eingeflossen. Die Schlussfassung des Beitrags profitiert von den konstruktiven Anregungen zweier anonymer GutachterInnen und von Sören Becker.

Edited by: S. Becker

Reviewed by: two anonymous referees

\section{Literatur}

Adorno, Th. W. und Horkheimer, M.: Dialektik der Aufklärung, Philosophische Fragmente, Suhrkamp, Frankfurt a.M., 1947.

Bachram, H.: Climate Fraud and Carbon Colonialism: The New Trade in Greenhouse Gases, Capitalism, Nature, Socialism, 15, 1-16, 2004.

Bakker, K.: Neoliberal nature, ecological fixes, and the pitfalls of comparative research, Environ. Plan. A, 41, 1781-1787, 2009.

Bakker, K. und Bridge, G.: Material worlds? Resource geographies and the "matter of nature", Prog. Hum. Geog., 30, 5-27, 2006.

Barad, K.: Posthumanist performativity: Toward an understanding of how matter comes to matter, Signs - J. Women in Culture Society, 28, 801-831, 2003.

Barad, K.: Meeting the Universe Halfway. Quantum Physics and the Entanglement of Matter and Meaning, Duke University Press, Durham, NC, 2007.

Bauriedl, S.: Eurozentrische Weltbürgerbewegung. Zum WBGUSondergutachten Klimaschutz als Weltbürgerbewegung, GAIA, 24, 13-16, 2015a.

Bauriedl, S.: Klimapolitik. Fortsetzung globaler und sozialer Ungleichheiten, Prokla, 181, 629-636, 2015 b.

Becker, E. und Jahn, Th. (Hrsg.): Soziale Ökologie. Grundzüge einer Wissenschaft von den gesellschaftlichen Naturverhältnissen, Campus, Frankfurt a.M., 2006.

Bennett, J.: Vibrant matter. A political ecology of things, Duke University Press, Durham, NC, 2010.

Blaikie, P. M.: The Political Economy of Soil Erosion in Developing Countries, Longman, Harlow Essex, 1985. 
Bohle, H.-G.: Neue Ansätze der geographischen Risikoforschung: Ein Analyserahmen zur Bestimmung nachhaltiger Lebenssicherung von Armutsgruppen, Die Erde, 132, 119-140, 2001.

Bohle, H.-G.: Geographien von Verwundbarkeit, Geog. Rundschau, 59, 20-25, 2007.

Brand, U. und Wissen, M.: Sozial-ökologische Krise und imperiale Lebensweise. Zu Krise und Kontinuität kapitalistischer Naturverhältnisse, in: VielfachKrise im finanzdominierten Kapitalismus, Herausgeber: Demirović, A., Dück, J., Becker, F. und Bader, P., VSA, Hamburg, 78-93, 2011a.

Brand, U. und Wissen, M.: Die Regulation der ökologischen Krise. Theorie und Empirie der Transformation gesellschaftlicher Naturverhältnisse, Österr. Z. Soziologie, 36, 12-34, 2011 b.

Braun, B.: From Critique to experiment. Rethinking political ecology for the Anthropocene, in: The Routledge Handbook of Political Ecology, Herausgeber: Perreault, Th. und Bridge, G., Routledge, London, New York, 102-114, 2015a.

Braun, B.: New Materialisms and Neoliberal Natures, Antipode, 47, 1-14, 2015b.

Bryant, R. L.: The international Handbook of Political Ecology, Edward Elgar Publishing, Cheltenham, UK, 2015.

Bryant, R. L. und Bailey, S.: Third World Political Ecology. An Introduction, Routledge, London, New York, 1997.

Castree, N.: Neo-liberalising nature II: Processes, outcomes and effects, Environ. Plann. A, 40, 153-173, 2008.

Chakrabarty, D.: Provincializing Europe. Postcolonial Thought and Historical Difference, Princeton University Press, Princeton, 2000.

Chakrabarty, D.: Postcolonial studies and the challenge of climate change, New Literary Hist., 43, 1-18, 2012.

Coole, D. und Frost, S.: Introducing the New Materialisms, in: New Materialisms. Ontology, Agency, and Politics, Herausgeber: Coole, D. und Frost, S., Duke University Press, Durham, London, 2010.

Dietz, K. und Engels, B.: Land Grabbing analysieren: Ansatzpunkte für eine politisch-ökologische Perspektive am Beispiel Äthiopiens, Peripherie, 124, 399-420, 2011.

Egner, H. und Pott, A. (Hrsg.): Geographische Risikoforschung: Zur Konstruktion verräumlichter Risiken und Sicherheiten, Franz Steiner Verlag, Stuttgart, 2010.

Ehrlich, P. R.: The Population Bomb, Ballantine Publisher, New York, 1968 (deutsch: Die Bevölkerungsbombe, Hanser, München, 1971).

Ekers M. und Loftus, A.: Revitalizing the production of nature thesis. A Gramscian turn?, Prog. Hum. Geog., 37, 234-252, 2012.

Enzensberger, H. M.: Zur Kritik der politischen Ökologie, Kursbuch 33, 1-42, 1974.

Fairhead, J. und Leach, M.: Misreading the African Landscape: Society and Ecology in a Forest-Savanna Mosaic, Cambridge University Press, Cambridge, 1996.

Fairhead, J., Leach, M. und Scoones, I.: Green Grabbing: a new appropriation of nature?, J. Peasant Stud., 39, 237-261, 2012.

Felgentreff, C. und Glade, Th. (Hrsg.): Naturrisiken und Sozialkatastrophen, Springer Verlag, Heidelberg, 2008.

Flitner, M.: Konstruierte Naturen und ihre Erforschung, Geogr. Helv., 53, 89-95, doi:10.5194/gh-53-89-1998, 1998.

Forsyth, T.: Politicizing environmental explanations: what can political ecology learn from sociology and philosophy of science?, in: Knowing Nature, Herausgeber: Goldman, M. J., Nadasdy, P. und Turner, M. D., University of Chicago Press, Chicago, 31-46, 2011.

Görg, Ch.: Gesellschaftliche Naturverhältnisse, Westfälisches Dampfboot, Münster, 1999.

Görg, Ch.: Regulation der Naturverhältnisse. $\mathrm{Zu}$ einer kritischen Theorie der ökologischen Krise, Westfälisches Dampfboot, Münster, 2003.

Görg, Ch.: Planetarische Grenzen, in: Wörterbuch Klimadebatte, Herausgeberin: Bauriedl, S., Transcript, Bielefeld, 239-244, 2016.

Grosfoguel, R.: The epistemic decolonial turn. Beyond politicaleconomy paradigms, Cultural Stud., 21, 211-223, 2007.

Harvey, D.: The "new" imperialism: accumulation by dispossession, Socialist Register, 40, 63-87, 2004.

Harvey, D.: Spaces of global capitalism: a theory of uneven geographical development, Verso, London, 2006.

Heynen, N., McCarthy, J., Prudham, S. und Robbins, P. (Hrsg.): Neoliberal Environments. False Promises and Unnatural Consequences, Routledge, London, New York, 2007.

Jackson, M.: Composing postcolonial geographies. Postconstructivism, ecology and overcoming ontologies of critique, Singapore J. Trop. Geog., 35, 72-87, 2014.

Keil, R., Bell, D., Penz, P. und Fawcett, L.: Political Ecology: Global and Local, Routledge, London, New York, 1998.

Krings, Th. und Müller, B.: Politische Ökologie. Theoretische Leitlinien und aktuelle Forschungsfelder, in: Politische Geographie. Handlungsorientierte Ansätze und Critical Geopolitics, Herausgeber: Reuber, P. und Wolkersdorfer, G., Heidelberger Geographische Arbeiten, Heidelberg, 93-116, 2001.

Leach, M., Rockström, J., Raskin, P., Scoones, I., Stirling, A. C., Smith, A., Thompson, J., Millstone, E., Ely, A., Arond, E., Folke, C. and Olsson, P.: Transforming innovation for sustainability, Ecol. Soc., 17, 11-15, 2012.

Lorimer, J.: Ladies and Gentlemen, behold the enemy!, Environ. Plan. D, 28, 40-42, 2010.

Malthus, Th. R.: An Essay on the Principle of Population, www.econlib.org/library/Malthus/malPop.html (letzter Zugriff: 29. Mai 2016), 1789.

McCarthy, J. und Prudham, S.: Neoliberal nature and the nature of neoliberalism, Geoforum, 35, 275-283, 2004.

McEwan, Ch.: Material geographies and postcolonialism, Singapore J. Trop. Geog., 24, 340-55, 2003.

MEA - Millennium Ecosystem Assessment: Ecosystems and $\mathrm{Hu}-$ man Well-being, Synthesis, Island Press, Washington, DC, 2005.

Meadows, D., Meadows, D. H., Randers, J. und Behrens, W. W.: Die Grenzen des Wachstums. Bericht des Club of Rome zur Lage der Menschheit, DVA, Stuttgart, 1972.

Mignolo, W. D.: The Darker Side of Western Modernity. Global Futures, Decolonial Options, Duke University Press, Durham, NC, 2011.

Moore, J.: Nature in the limits to capital (and vice versa), Radical Philos., 193, 9-19, 2015.

Müller-Mahn, D. (Hrsg.): The Spatial Dimension of Risk: How Geography Shapes the Emergence of Riskscapes, Routledge, London, New York, 2012.

Neumann, R.: Making Political Ecology, Hodder Arnold, New York, 2005.

Peet, R.: Social origins of environmental determinism, Annals Ass. Am. Geog., 75, 309-333, 1985. 
Peet, R. und Watts, M.: Liberation Ecology: Environment, Development, Social Movements, Routledge, London, New York, 1996.

Peet, R., Robbins, P. und Watts, M.: Global Political Ecology, Routledge, London, New York, 2010.

Perreault, Th. und Bridge, G.: The Routledge Handbook of Political Ecology, Routledge, London, New York, 2015.

Radcliffe, S. A.: Development and geography: towards a postcolonial development geography?, Prog. Hum. Geog., 29, 291-298, 2005.

Robbins, P.: Political Ecology. Critical Introduction to Geography, Wiley-Blackwell, Chichester, 2004.

Rockström, J., Steffen, W., Noone, K., Persson, Å., Chapin, S., Lambin, E. F., Lenton, T. M., Scheffer, M., Folke, C., Schellnhuber, H. J., Nykvist, B., de Wit, C. A., Hughes, T., van der Leeuw, S., Rodhe, H., Sörlin, S., Snyder, P. K., Costanza, R., Svedin, U., Falkenmark, M., Karlberg, L., Corell, R. W., Fabry, V. J., Hansen, J., Walker, B., Liverman, D., Richardson, K., Crutzen, P. und Foley, J. A.: A safe operating space for humanity, Nature, 461, 472-475, 2009.

Smith, N.: Uneven Development. Nature, Capital and the Production of Space, Oxford University Press, Oxford, 1984.

Steffen, W., Crutzen, P. und McNeill, J. R.: The Anthropocene: Are Humans Now Overwhelming the Great Forces of Nature?, Ambio, 36, 614-621, 2007.
Swyngedouw, E.: Social Power and the Urbanization of Water. Flows of Power, Oxford University Press, Oxford, 2004.

Swyngedouw, E.: The Non-political Politics of Climate Change, ACME: Intern. J. Crit. Geog., 12, 1-8, 2013.

Turner, M. D.: Political Ecology and its Engagements with Conservation and Development, ACME: Intern. J. Crit. Geog., 13, 478-488, 2014.

Watts, M.: Silent Violence: Food, Famine, and Peasantry in Northern Nigeria, University of California Press, Berkeley, 1983.

Watts, M.: Political ecology, in: A Companion to Economic Geography, Herausgeber: Barnes, T. und Sheppard, E., Blackwell, Oxford, 257-275, 2000.

Whatmore, S.: Hybrid Geographies: Natures Cultures Spaces, Sage, London, 2002.

Whatmore, S.: Political Ecology in a More-than-Human World: Rethinking "Natural" Hazards, in: Anthropology and Nature, Herausgeber: Hastrup, K., Routledge, London, New York, 7995, 2013.

Wisner, B.: Speaking truth to power. A personal account of activist political ecology, in: The Routledge Handbook of Political Ecology, Herausgeber: Perreault, Th. und Bridge, G., Routledge, London, New York, 53-63, 2015.

Wolf, E.: Ownership and Political Ecology, Anthropological Quart., 45, 201-205, 1972. 